\title{
Salutogenesis Beyond Health: Intergroup Relations and Conflict Studies
}

\author{
Shifra Sagy and Adi Mana
}

\section{Introduction}

Antonovsky's salutogenic model and its core concept "sense of coherence" (SOC) focus on the ability of individuals to cope with stressors in life and stay healthy. Accordingly, the relationship between SOC and health has received much attention and quite consistent results in research (see Chap. 9 of this book). However, since the paradigm of salutogenesis was introduced within the discipline of sociology of health, the relationships between salutogenesis and other social concepts have been mostly neglected. Unfortunately, until recently, the salutogenic model has almost never been broadened into an interdisciplinary framework. We believe that, at this stage of its development and its wide distribution in the scientific world, the salutogenic paradigm should broaden its scope. In proposing broadening the application of salutogenesis to interdisciplinary research, we may consider disciplines like social psychology, economics, geography, anthropology, or conflict studies. This chapter aims to raise new questions about applying the salutogenic paradigm to research in interdisciplinary fields and to review new studies that have already begun to do so.

This chapter is a revision and update of work published in Mittelmark, M.B., Sagy, S., Eriksson, M., Bauer, G., Pelikan, J.M., Lindström, B., and Espnes, G.A. (eds). (2017). The Handbook of Salutogenesis. Springer, Cham. DOI: https://doi.org/10.1007/978-3-319-04600-6.

S. Sagy $(\bowtie)$

Martin Springer Center for Conflict Studies, Ben-Gurion

University of the Negev, Be'er Sheva, Israel

e-mail: shifra@bgu.ac.il

A. Mana

School of Behavioral Science, Peres Academic Center, Rehovot, Israel

\section{Dilemmas in Broadening Salutogenesis into an Interdisciplinary Framework}

Broadening salutogenesis into an interdisciplinary framework raises several core dilemmas. First, we must deal with the questions: Does the salutogenic orientation enable us to deal with other concepts beyond the relationship with health? And if so, how? How can we ask salutogenic questions employing concepts embedded in other disciplines?

Let's start with the first question. In his writing about the development of SOC, Antonovsky wrote extensively about how one's life experiences can influence the strength of one's SOC (Antonovsky, 1987). Actually, in his dealing with this issue - the origins of SOC - Antonovsky expressed an interdisciplinary approach by relating to broad-ranging factors like culture, social forces, social position, gender, ethnicity, genetics, or even plain luck (Benz et al., 2014). Indeed, social factors have seldom been studied as predictors of SOC (e.g., Lam, 2007; Sagy \& Antonovsky, 2000). Two aspects have mainly been studied: the experience of cultural integration vs discrimination due to being part of a minority group (Ying et al., 2001) and the experience of cultural stability vs instability (e.g., Antonovsky \& Sagy, 1986).

Even though this body of research was mostly composed of correlational studies, which leave the direction of causality undetermined, in most of the studies, the suggested direction was that elements related to a variety of social factors should be considered as predictors of SOC.

However, while Antonovsky discussed the other direction of the equation, meaning how SOC can influence life situations, his answer was much less interdisciplinary. He clarified in his work that "a salutogenic orientation, no less than a pathologic one, defines health and disease only in terms of functioning and survival. All that it argues is that the stronger the SOC, the more likely the system, whether individual, family, or society, to function and survive" (Antonovsky, 1991, p. 8). What he meant was that SOC, whose development is influenced by social factors, cannot predict social concepts, which could have a positive or negative connotation. 
Moreover, Antonovsky viewed such social factors as loaded by moral-philosophical problems and as such not inherently within the context of salutogenesis (Antonovsky, 1991). This original conviction of his can partly explain the focus of salutogenic research over almost four decades on the SOC/ health hypothesis and why it has not been broadened to include other interdisciplinary concepts as well.

By raising this question, once again, we assume that salutogenesis, 40 years later, is challenged by the call of interdisciplinarity. We believe that researchers can ask salutogenic questions in different areas, not only health and well-being, and perhaps find other salutogenic answers. When we employ an interdisciplinary approach, the salutogenic question would not only be "Who copes successfully and stays healthy?" (Antonovsky, 1987) but also, for example, "Who expresses more openness to the other?", "Who is a social activist who pursues justice in the world"? Or "Who is a peacemaker?" If we broaden the level of our questions, we can ask about salutogenic schools, neighborhoods, or other social institutions, which enhance not only health and wellness but also justice, peace, and reconciliation. The definitions of these concepts can vary over different contexts and cultures, but the question remains a salutogenic one.

\section{Toward Exploring Salutogenic Questions in an Interdisciplinary Framework}

Now we arrive at the second part of our review: How can we ask salutogenic questions in an interdisciplinary framework? Relating to this "how" question, we will review some studies, which have already attempted to do so. Previous studies found positive interactions between SOC and the relationships between members of the same community (e.g., for reviews, see Koelen \& Eriksson, and Vaandrager \& Kennedy in this handbook; Maass et al., 2014; Morton \& Lurie, 2013; Teig et al., 2009). This evidence was explained by the well-known relationship between strong social connection or connectivity and enhanced sense of health and well-being (Vaandrager \& Kennedy, 2017). However, this explanation brings us back to the SOC/health equation, and we are looking for other equations as well.

Thus, in our attempt to move beyond the SOC/health equation, we decided to explore other equations like $\mathrm{SOC} /$ social relations or SOC/political orientations. Several studies have attempted to examine the relationship between SOC and personal traits which could facilitate social relations with out-group members. Feldt et al. (2007) analyzed the relations between SOC and the five-factor model of personality. The results indicate that a person with a strong SOC shows modest positive associations with extraversion, openness, conscientiousness, and agreeableness. Another study (Pålsson et al., 1996) found negative correlations between SOC and the personality traits of avoidance, detachment, hostility, and aggression and positive correlations between SOC and empathy.

In political psychology, SOC was measured as a predictor of different political orientations. No correlations were found, for example, between SOC and attitude scales measuring patriotism, nationalism, and authoritarianism (Renner et al., 2004). However, political attitudes toward peace were found in correlation with strong SOC (Braun-Lewensohn et al., 2015 ) in a study of Israeli adolescents during a political, violent event, in the context of the Israeli-Palestinian conflict. Adolescents who had a strong SOC had a strong tendency to view the conflict as another challenge in life with which to cope and perceived the conflict as manageable and as meaningful. This study, however, was a one-time, cross-sectional study and its causal interpretation could also be different.

The findings related to the equations of SOC/social relations or SOC/political orientation are quite ambiguous. We trust that more studies should be carried out to support their promising results. The prominent direction revealed in this body of research is that a strong SOC is connected with tendencies associated with positive values, at least in Western society. Are these conclusions that Antonovsky tried to avoid? Perhaps yes. In his lecture in Prague in 1991, he warned of the danger of defining health so that it becomes "...a catchall for anything that you think is good. Health then becomes not a scientific concept, but confused with a set of answers to moral-philosophical problems...the distinction must be made" (Antonovsky, 1991 p.9). Of course, when we broaden salutogenesis to include concepts other than health, we cannot avoid these moral-philosophical questions about values and science. Thus, we have to deal with them while fully recognizing our limitations in making such a distinction.

\section{Sense of Coherence: From the Individual to the Collective Concept}

Since attitudes and behaviors toward the other are developed within one's social context, the relationship between SOC and intergroup relations should be explored not only from the individual perspective but also in the supra-system context (Sagy, 2014, 2017). The global perception of the world as coherent does not necessarily mean that people must perceive the entire group they belong to as comprehensible, manageable, and meaningful. Relating to this question Antonovsky suggested: "Quite conceivably, people might feel that they have little interest in national government or international politics, little competence in manual (or cognitive or aesthetic) skills, little concern for local volunteer groups or trade union activity, and so on, and yet have a strong SOC" (Antonovsky, 1984, p. 119). 
Van Breda (2001), in his review, based on the concept of "circle of concern and influence" (Covey \& Merrill, 2006), explained that people draw boundaries within the objective world - provided those things which fall within the boundaries are considered coherent - the person will have a strong SOC, irrespective of the coherence of things outside the boundaries. Things outside of the circle of concern can be of no importance to that individual. Within the circle of concern is a smaller circle, the "circle of influence," which refers to those things which concern that individual and over which that individual has some influence. By focusing on the area between the two circles (i.e., those things that concern one but over which one has no influence), one creates SOC-reducing experiences, since the situation is not manageable. By focusing on issues within the circle of influence, one is assured of life experiences that are coherent, and in so doing, one can (theoretically) expand the circle of influence. There are cases when a person may have a very small circle of concern and an even smaller circle of influence, yielding a life that is very limited in scope, although potentially high in SOC. This question of boundaries within the objective world brings us to our second branch of research that deals with the role of sense of coherence in intergroup relationships.

The idea that the SOC concept should be broadened to larger levels than individuals has been suggested and discussed by Sagy and Antonovsky (Antonovsky, 1992; Sagy, 1990; Sagy \& Antonovsky, 1992). It is beyond the scope of this chapter, however, to include this extensive discussion. Here, we will review some studies that link the SOC of the collective to intergroup and social relations.

\section{Sense of Community Coherence}

The concept of sense of community coherence (SOCC) was developed as related to a specific in-group and not to the "global orientation of the world" as it is defined for individual SOC. It constitutes the three components of SOC: comprehensibility, manageability, and meaningfulness (Braun-Lewensohn \& Sagy, 2011; Elfassi et al., 2016; Peled et al., 2013; Sagy, 1998; Telaku et al., 2020). Community comprehensibility relates to the perception that life in one's community is predictable, safe, and secure and that one's community is a place that is known and understood. Community manageability relates to the perception that one's community can assist its members, is available to them, and meets their demands and needs. Lastly, community meaningfulness relates to the perception that the community gives meaning to its members, provides challenges, and is worthy of investment and engagement.

Most of the research which investigated the concept of SOCC has focused on its relationship with well-being and mental health, meaning the SOC/health equation. For example, positive relations have been found between a strong sense of community coherence and the level of resilience to stressful events (bomb attacks, a fire disaster) (e.g., BraunLewensohn et al., 2013; Braun-Lewensohn \& Sagy, 2011). SOCC was found as negatively related to unhealthy behaviors. Elfassi et al. (2016) found significant negative correlations between SOCC and the levels of risk behaviors among Israeli adolescents and claimed that SOCC is a significant protective factor that could be related to decreased involvement in risk behaviors.

Just recently, however, several studies have attempted other equations, seeking to connect salutogenesis and SOC to other social concepts like intergroup relations, openness toward the other, and readiness to reconcile (Mana et al., 2019; Sagy, 2014; Sagy, 2017; Srour, 2015; Telaku et al., 2020). These studies connect the salutogenic paradigm with interdisciplinary models and concepts like social identity (Tajfel, 1981), acculturation (Berry, 1990), conflict studies (Bar-Tal, 1998), or peace and reconciliation (Nadler, 2012). The interdisciplinary salutogenic questions in these studies are: How does a collective with a strong SOC perceive, feel, or behave toward the other? Is the tendency of a group to perceive its world as comprehensible, meaningful, and manageable related to greater openness to the other, or does it involve clinging to rigid in-group identity and less openness toward the other? Is an individual, a group, a collective, or a system with a stronger SOC more likely to live in peace/ justice/good relations with their surroundings?

Most of these new studies examined the relationship between SOCC and intergroup relations. The relations between the conflicted groups were examined by the levels of adherence to in-group collective narratives as well as acceptance of the out-group collective narratives. Some of the studies found a relationship between SOCC and acculturation attitudes (Somech \& Sagy, 2019; Telaku \& Sagy, 2018). One of the studies was conducted among Palestinian Muslims and Christians in Israel (Mana et al., 2016).

The results revealed that strong sense of community coherence (SOCC) was correlated with higher levels of acceptance of the in-group collective narrative and with lower levels of acceptance of the out-group collective narratives. Sense of community coherence was also related to higher levels of a tendency to adopt a separation strategy between the two groups in conflict.

The explanations for all these studies relied on a wide range of models in social psychology which suggest that group members who believe that their own group and its products are superior to other groups are prone to behaviorally discriminate against other groups (e.g., Bizumic \& Duckitt, 2009). This notion was well established in the work of Tajfel (1981) who analyzed three cognitive aspects of prejudice: 
the process of categorization, which gives shape to intergroup attitudes, the process of assimilation of social values and norms which provides their content, and as a main cognitive aspect which relates to the way individuals react to specific intergroup situations.

Following Tajfel's paradigm, we can interpret the strong SOCC as enabling group members to deal with changes that occur in intergroup situations. In order to deal with these changes, an individual must make constant attributions that help him deal with the new situations in a manner that appears consistent to him and preserves his self-image and integrity. The individual needs to build a cognitive structure which provides him with a satisfactory explanation of the causes of changes.

However, an improved group position is often achieved by using the group's capacity to put another group at a disadvantage and derives largely from biased comparisons on salient dimensions that are favorable to the in-group and unfavorable to the out-group (Brown, 2000; Tajfel, 1981).

Several studies focused on SOCC in the context of interreligious group relations and international group relations in different social contexts. One was carried out in the context of two religious communities in Israel: ultra-Orthodox and national religious Jews (Somech \& Sagy, 2019).

The results of this study indicated the same tendency of connection with openness to the other, while the ultraOrthodox community exhibited stronger SOCC than their counterparts, as well as a stronger connection of SOCC with separation tendencies. Mana et al. (2020) suggested a mediation model in which the relationships between SOCC and the identity strategies of separation and competition are mediated by the tendency to reject or accept in-group and out-group collective narratives, in the context of intra- and inter-religious conflicts.

They compared the intra-religious conflict between ultraOrthodox Jews and national religious Jews and the interreligious conflict between Muslim and Christian Arabs who are Israeli citizens and found that the mediation model was stronger among those groups with a high SOCC (ultraOrthodox Jews and Muslim Arabs) compared to groups with low SOCC (Christian Arabs and national religious Jews).

Another study (Telaku et al., 2020) related to SOCC and intergroup relations in the context of post-armed conflict between Serbs and Albanians in Kosovo. This study suggested that another factor - subjective experience of interpersonal power - mediates the relationships between SOCC and openness to the other, as measured by perceptions of collective narratives of the out-group and the in-group. The model was confirmed among both Serb and Albanian participants.

This review indicates that sense of coherence at the community level is indeed a salutogenic and health-promoting factor that has a significant role in the ability of community members to cope with threatening situations on the collective level (SOC/health equation). At the same time, however, the studies suggest SOCC as a barrier for positive intergroup relationships in different kinds of conflicts: intractable or periodic, political, ethnic, or religious conflicts.

In all of these conflicts, it appears that SOCC leads the community members to adhere to their own collective narratives and to reject the collective narratives of the outgroup and to adopt strategies of separation from the out-group members. Although this process was explored as a linear process, it seems that it could also be circular, and the tendency of the in-group members to prefer their own group members, and their own collective narratives, while rejecting the others, may contribute to their perception of their own community as more comprehensive, manageable, and meaningful. Unfortunately, it seems that this pattern has a long-lasting effect even when the armed conflict is over, like in Kosovo. However, the preliminary evidence revealed that this pattern could be affected by other mediating factors like the subjective experience of interpersonal power. It has also been found to be more effective among groups with stronger SOCC. Exploring individual and group differences that enable group members to act differently could promote new insights to "break this chain" of the contribution of strong SOCC as a barrier to the possibility of openness to the other group and readiness to reconcile in intergroup relationships.

\section{Sense of National Coherence}

A wider concept recently suggested by Sagy (2014) relates to the national level, the sense of national coherence (SONC). The salutogenic concept of SONC reflects an enduring tendency to perceive one's national group as comprehensible, meaningful, and manageable.

The main assumption of the researchers who examined this concept is that SONC can provide a deeper understanding of political conflict situations. As such, SONC could be a resource of wellness and health, but, at the same time, it could serve as a potential barrier for a peace process in a conflict area. The first studies related to SONC were conducted in the context of the Israeli-Palestinian intractable conflict (Mana et al., 2020; Sarid et al., in press; Sarid \& Sagy, 2015).

The initial results indicate a strong negative correlation between strong SONC and levels of openness to the narrative of the other group. Moreover, levels of SONC among Israeli students before and after a violent period (the Gaza war) indicated an increase in the level of SONC and a significant decrease in willingness to reconcile. The negative correlation between those two variables was stronger after the Gaza war than before it. 
The findings suggest that the escalation in the conflict and in violence has an impact on the perceptions of the national sense of coherence and could be a potential barrier to reconciliation. Mana et al. (2019) explored SONC and its relationship with openness to the Palestinian collective narrative among Israeli-Jewish adults. SONC was found to be negatively related to level of legitimization toward the Palestinian collective narratives. This finding replicated previous studies related to SOCC. Moreover, SONC was found to be related to right-wing voting patterns and to religious groupings (Mana et al., 2019).

Research on SONC has moved in different directions beyond the political sphere to educational initiatives. For example, in a work submitted for publication, Agbaria, Mana, Bar-Gera, and Sagy et al., investigated levels of SONC with the aim of explaining behavioral intentions toward driving among young Israeli Jews and Arabs. The findings indicate strong correlations between SONC, behavioral intentions, and readiness to take risks while driving, and to the perceptions of the police as representing justice, especially among the minority group of Arab adolescents in Israel. The results suggest some implications for educational interventions among youngsters before their driving experience.

\section{Salutogenesis and the COVID-19 Pandemic: Sociopolitical Considerations}

We are writing this chapter in the midst of the COVID-19 pandemic, which has shaken up lives worldwide and is having strong economic, social, and political impacts in many countries. Naturally, the pandemic has led researchers to ask pathogenic questions about its pathogenic origins and the potential damage. This chaotic global pandemic, however, was also an opportunity to explore salutogenic concepts like SOC and SONC and to understand their roles not only in promoting health but also beyond health, as potential factors in increasing some order out of the chaos. Mana and Sagy, jointly with the Global Working Group on Salutogenesis, conducted some international research (Mana, Bauer, et al., 2021; Hardy et al., 2021; Mana, Super et al., 2021; Mana \& Sagy, 2020). The theoretical basis of the salutogenesis, and its fundamental philosophical postulate that the world is chaotic in its nature, was an appropriate basis for these studies.

We revealed the important role of the salutogenic concepts of SOC and SONC in predicting higher levels of mental health and lower levels of anxiety in all of the countries involved in our research from Europe, Israel, and the USA. Beyond this, we sought to understand the socialpolitical situation that has been developed while the acute stressor of the pandemic has become chronic. SONC, for example, was found to be highly related to level of trust in governmental institutions.

This relationship was even stronger among people who voted for the current government (Mana \& Sagy, 2020; Super et al., 2020). However, low SONC, as well as low trust in the leadership, was strongly connected to lower levels of mental health. Our preliminary findings reveal that levels of SONC also decreased as the pandemic moved from acute to chronic stress, and the political stability in many countries has been eroded.

These studies could deepen our understanding of the theoretical basis of the salutogenesis. Moreover, these current studies reveal the relationships between sense of coherence at the personal and the collective levels and their role not only in the SOC/health equation but also in social relationships and political processes in the context of the chronic stress, which introduces entropy into the global system.

In conclusion, while there is a broad consensus regarding the contribution of SOC to health and well-being, the role of SOC - both of the individual or the collective - in social relations has been mostly neglected. We maintain that one of the reasons for this neglect is the initial excitement of Antonovsky and his followers about studying the SOC/ health connection. Times have changed and interdisciplinarity seems to be the challenge of our era. In this chapter, we have attempted to review the small body of research, which asks other salutogenic questions about the relationship of SOC at the individual and the collective levels to other interdisciplinary concepts.

We believe that more research is needed in order to gain a deeper understanding of these initial answers. Moreover, interdisciplinarity can also lead to employing other salutogenic concepts - rather than SOC - to give answers to salutogenic questions. So we suggest that salutogenic researchers in the future not only ask new salutogenic questions but also develop new salutogenic concepts in the attempt to broaden and deepen our understanding of the paradigm. We hope that this chapter succeeds in posing this new challenge.

\section{References}

Agbaria, K., Mana, A., Bar-Gera, H., \& Sagy, S. (Submitted). Personal and social factors related to behavioral intentions towards driving among teenagers: Comparing Israeli Arabs and Jews.

Antonovsky, A. (1984) The sense of coherence as a determinant in health. In: J.D. Matarazzo, (Ed.). Behavioral health: A handbook of health enhancement and disease prevention, (pp. 114-129) John Wiley.

Antonovsky, A. (1987). Unraveling the mystery of health. Jossey-Bass. Antonovsky, A. (1991). The Salutogenic approach to family system health: Promise and danger. Lecture at European Congress on "Mental Health in European Families", Prague, Czechoslovakia, 
5-8 May 1991. Retrieved from: http://www.angelfire.com/ok/soc/ agolem.html.

Antonovsky, A. (1992). Can attitudes contribute to health? Advances, 8, 33-49.

Antonovsky, H., \& Sagy, S. (1986). The development of a sense of coherence and its impact on responses to stress situations. Journal of Social Psychology, 126, 213-227.

Bar-Tal, D. (1998). Social beliefs in times of intractable conflict: The Israeli case. International Journal of Conflict Management, 9, 22-50.

Berry, J. W. (1990). Psychology of acculturation. In J. J. Berman (Ed.), Nebraska symposium on motivation, 1989: Cross-cultural perspectives. Current theory and research in motivation, 37 (pp. 201-235). University of Nebraska Press.

Benz, C., Bull, T., Mittelmark, M., \& Vaandrager, L. (2014). Culture in salutogenesis: The scholarship of Aaron Antonovsky. Global Health Promotion., 21(4), 16-23.

Bizumic, B., \& Duckitt, J. (2009). Narcissism and ethnocentrism: A review. Directions in Psychiatry, 29, 99-109.

Braun-Lewensohn, O., Abu-Kaf, S., \& Sagy, S. (2015). Attitudes toward war and peace and their relations with anxiety reactions among adolescents living in a conflictual area. Journal of Youth Studies, 18(1), 68-79.

Braun-Lewensohn, O., \& Sagy, S. (2011). Salutogenesis and culture. Personal and community sense of coherence among adolescents belonging to three different cultural groups. International Review of Psychiatry, 23(6), 533-541.

Braun-Lewensohn, O., Sagy, S., Sabato, H., \& Galili, R. (2013). Sense of coherence and sense of community as coping resource of religious adolescents before and after the disengagement from the Gaza strip. Israeli Journal of Psychiatry \& Related Sciences, 50(2), $110-117$

Brown, R. (2000). Social identity theory: Past achievements, current problems and future challenges. European Journal of Social Psychology, 30(6), 745-778. https://doi.org/10.1002/10990992(200011/12)30:6<745::AID-EJSP24>3.0.CO;2-O

Covey, S. M. R., \& Merrill, R. R. (2006). The speed of trust: The one thing that changes everything. CoveyLink.

Elfassi, Y., Braun-Lewensohn, O., Krumer-Nevo, M., \& Sagy, S. (2016). Community sense of coherence among adolescents as related to their involvement in risk behaviors. Journal of Community Psychology, 44(1), 22-37. https://doi.org/10.1002/jcop.21739

Feldt, T., Metsäpelto, R.-L., Kinnunen, U., \& Pulkkinen, L. (2007). Sense of coherence and five-factor approach to personality: Conceptual relationships. European Psychologist, 12(3), 165-172.

Hardy, L., Mana, A., Mundell, L., Benheim, S., Torres Morales, K., \& Sagy, S. (2021). Living in opposition: How women in the United States cope in spite of mistrust of federal leadership during the pandemic of COVID-19. Journal of Community Psychology. 2021 Mar 17. https://doi.org/10.1002/jcop.22544. Epub ahead of print. PMID: 33729586.

Lam, B. T. (2007). Impact of perceived racial discrimination and collective self-esteem on psychological distress among VietnameseAmerican college students: Sense of coherence as mediator. American Journal of Orthopsychiatry, 77(3), 370-376.

Maass, R., Lindström, B., \& Lillefjell, M. (2014). Exploring the relationship between perceptions of neighborhood-resources, sense of coherence and health for different groups in a Norwegian neighbourhood. Journal of Public Health Research, 3(208).

Mana, A., Bauer, A., Meier Magistretti, C., Sardu, C., Juvinyà Canal, D., Hardy, L.J., Catz, O., Tušl, M., \& Sagy, S. (2021). Order out of chaos: Sense of coherence and the mediating role of coping resources in predicting mental health during COVID-19 in 7 countries. SSM \& Mental Health, 1. Online Advanced https://doi. org/10.1016/j.ssmmh.2021.100001.
Mana, A., \& Sagy, S. (2020). Brief report: Can political orientation explain mental health in the time of a global pandemic? Voting patterns, personal and national coping resources, and mental health during the coronavirus crisis. Journal of Social and Clinical Psychology, 39(3), 187-193. https://doi.org/10.1521/jscp.2020.39.3.165

Mana, A., Sagy, S., \& Srour, A. (2016). Sense of community coherence and inter-religious relations. The Journal of Social Psychology, 156(5), 469-482. https://doi.org/10.1080/00224545.2015.1129302

Mana, A., Sagy, S., \& Srour, A. (2019). Sense of National Coherence and openness to the "other's" collective narratives: The case of the Israeli-Palestinian conflict. Peace and Conflict: Journal of Peace Psychology, 25(3), 226-233. https://doi.org/10.1037/pac0000391

Mana, A., Srour, A., \& Sagy, S. (2020). Sense of community coherence, perceptions of collective narratives, and identity strategies in intraand inter- religious group conflicts. In A. Mana \& A. Srour (Eds.), Israeli and Palestinian collective narratives in conflict: A tribute to Shifra Sagy and her work (pp. 131-157). Cambridge Scholars.

Mana, A., Super, S., Sardu, C., Juvinya Canal, D., Neuman, M., \& Sagy, S. (2021). Individual, social and national coping resources and their relationships with mental health and anxiety: A comparative study in Israel, Italy, Spain, and the Netherlands during the Coronavirus pandemic. Global Health Promotion. 18:1757975921992957. https://doi.org/10.1177/1757975921992957. Epub ahead of print. PMID: 33601955; PMCID: PMC7897541.

Morton, M. J., \& Lurie, N. (2013). Community resilience and public health practice. American Journal of Public Health., 103(7), $1158-1160$.

Nadler, A. (2012). Intergroup reconciliation: Definition, processes and dilemmas. In L. Tropp (Ed.), Oxford handbook of conflict. Oxford University Press.

Pålsson, M. B., Hallberg, I. R., Norberg, A., \& Björvell, H. (1996). Burnout, empathy and sense of coherence among Swedish district nurses before and after systematic clinical supervision. Scandinavian Journal of Caring Science, 10(1), 19-26.

Peled, D., Sagy, S., \& Braun-Lewensohn, O. (2013). Community perceptions as coping resource among adolescents living under rockets fire: A salutogenic approach. Journal of Community Positive Practices, 4, 681-702.

Renner, W., Salem, I., \& Alexandrowicz, R. (2004). Human values as predictors for political, religious and health-related attitudes: A contribution towards validating the Australian value questionnaire (AVQ) by structural equation modeling. Social Behavior and Personality, 32(5), 477-490.

Sagy, S. (1990). The family sense of coherence and adjustment to stressors. Unpublished doctoral dissertation. Ben-Gurion University of the Negev.

Sagy, S. (1998). Effects of personal, family, and community characteristics on emotional reactions in a community stress situation: The Golan Heights negotiations. Youth \& Society, 29(3), 311-329.

Sagy, S. (2014). Rak al atzmi l' saper yadati: M'yomana shel hokeret sichsuchim b'heder hamugan. (only about myself did I know to tell: From the diary of a conflict researcher). Mifgash: Journal of social-educational Work, 40, 9-26. (Hebrew). http://www.jstor.org/ stable/24524253.

Sagy, S. (2017). Can we empathize with the narrative of our enemy? A personal odyssey in studying peace education. Intercultural Education, 28(6), 1-11. https://doi.org/10.1080/14675986.2017.1 390889

Sagy, S., \& Antonovsky, A. (1992). The family sense of coherence and the retirement transition. Journal of Marriage and the Family, 54, 983-993.

Sagy, S., \& Antonovsky, H. (2000). The development of the sense of coherence: A retrospective study of early life experiences in the family. International Journal of Aging \& Human Development, 51, $155-166$. 
Sarid, A., \& Sagy, S. (2015). National sense of coherence and openness to the other. Conference of Kibbutz studies.

Sarid, A., Srour, A., \& Sagy, S. (in press). Sense of national coherence and willingness to reconcile: The case of the Israeli- Palestinian conflict. In F. Ferrari, M., Leiner, Z. Barakat, \& M. Sternberg (Eds.), Encountering the Suffering of the Other. Transdisciplinary Perspectives from the DFG Project "Hearts of Flesh-not Stone" Vandenhoeck and Ruprecht.

Somech, L., \& Sagy, S. (2019). Perceptions of collective narratives and identity strategies as indicators of intergroup relations: The case of ultra-orthodox and National-Religious Jewish communities in Israel. International Journal of Conflict Management, 30(3), 290308. https://doi.org/10.1108/ijcma-06-2017-0058

Srour, A. (2015). Community sense of coherence, collective narrative perceptions and openness to the "other" group: The case of Muslims-Christians relations in Israel. PhD thesis. Ben Gurion University of the Negev (Hebrew).

Super, S., Pijpker, R., \& Polhuis, K. (2020). The relationship between individual, social and national coping resources and mental health during the COVID-19 crisis in the Netherlands. Health Psychology Report. https://doi.org/10.5114/hpr.2020.99028

Telaku, M., \& Sagy, S. (2018). Community sense of coherence and openness towards the other group: The case of Albanians and Serbs in Kosovo. In L. Gardner-Feldman, R. Barash, \& S. Goda (Eds.), Societies in transition: The former Soviet Union and east Central Europe between conflict and reconciliation. Vandenhoeck \& Ruprecht. The Jena Center for Reconciliation Studies' (JCRS) series on research in peace and reconciliation (RIPAR).
Telaku, M., Mana, A., Srour, A., \& Sagy, S. (2020). Sense of community coherence and perceptions of collective narratives in post-conflict context: The mediating role of subjective experience of interpersonal power. Peace and Conflict: Journal of Peace Psychology. https://doi.org/10.1037/pac0000525

Tajfel, H. (1981). Human groups and social categories: Studies in social psychology. Cambridge University Press.

Teig, E., Amulya, J., Bardwell, L., Buchenau, M., Marshall, J. A., \& Litt, J. S. (2009). Collective efficacy in Denver, Colorado: Strengthening neighborhoods and health through community gardens. Health \& Place, 15(4), 1115-1122. http://dx.doi.org/doi:\%2010.1016/\%20j. healthplace.2009.06.003

Vaandrager, L., \& Kennedy, L. (2017). Communities and neighborhoods. In M. Mittelmark, S. Sagy, M. Eriksson, G. F. Bauer, J. M. Pelikan, B. Lindström, \& G. A. Espnes (Eds.), Handbook of Salutogenesis (pp. 159-170). Springer International. https://link. springer.com/chapter/10.1007/978-3-319-04600-6_17

Van Breda, A. D. (2001). Resilience theory: A literature review (research report MPI/R/104/12/1/4). South African Military Psychological Institute. http://vanbreda.org/adrian/resilience/resilience_theory_ review.pdf.

Ying, Y. W., Lee, P. A., Tsai, J. L., Hung, Y., Lin, M., \& Wan, C. T. (2001). Asian American college students as model minorities: An examination of their overall competence. Cultural Diversity \& Ethnic Minority Psychology, 7(1), 59-74.

Open Access This chapter is licensed under the terms of the Creative Commons Attribution 4.0 International License (http://creativecommons. org/licenses/by/4.0/), which permits use, sharing, adaptation, distribution and reproduction in any medium or format, as long as you give appropriate credit to the original author(s) and the source, provide a link to the Creative Commons license and indicate if changes were made.

The images or other third party material in this chapter are included in the chapter's Creative Commons license, unless indicated otherwise in a credit line to the material. If material is not included in the chapter's Creative Commons license and your intended use is not permitted by statutory regulation or exceeds the permitted use, you will need to obtain permission directly from the copyright holder. 